\title{
Biological characteristics of Taxol-resistant ovarian cancer cells and reversal of Taxol resistance by adenovirus expressing p53
}

\author{
QUN LIU ${ }^{1}$, RUI SUI ${ }^{1}$, RUIRUI LI ${ }^{1}$, JINWEI MIAO ${ }^{1}$ and JIAN LIU ${ }^{2}$ \\ ${ }^{1}$ Department of Gynecologic Oncology, Beijing Obstetrics and Gynecology Hospital, Capital Medical University, \\ Beijing 100006; ${ }^{2}$ Medical Research Center, Beijing Chao-Yang Hospital, Capital Medical University, Beijing 100020, P.R. China
}

Received March 25, 2014; Accepted July 30, 2014

DOI: $10.3892 / \mathrm{mmr} .2014 .2784$

\begin{abstract}
The development of acquired drug resistance is the primary cause of chemotherapy failure in the treatment of ovarian cancer. To examine the mechanism underlying Taxol (TAX) resistance in ovarian cancer and attempt to reverse it, the present study induced a TAX-resistant ovarian cancer cell line SKOV3/TAX using a gradient concentration increment method. The properties of the resistant cell line were initially investigated by proliferation, colony formation, adhesion and cell cycle analysis compared with control SKOV3 cells. To examine the mechanism, the expression of p53 upregulated modulator of apoptosis (PUMA) was compared between SKOV3/TAX and SKOV3 cells by western blot analysis. An adenovirus expressing p53 (Ad-p53), alone or in combination with TAX, was used to treat the drug-resistant ovarian cancer cells SKOV3/TAX. The effects of Ad-p53 on pro-apoptosis and the reversal of drug resistance were evaluated using flow cytometric analysis, cleaved-poly ADP-ribose polymerase detection, microscopic observation and MTT measurement. Compared with the control cells, the TAX-resistant ovarian cancer cell line SKOV3/TAX was characterized by reduced sensitivity to TAX treatment, a significantly slower proliferation rate, higher colony-forming efficiency and higher adhesion ability. However, no significant difference in cell cycle distri-
\end{abstract}

Correspondence to: Dr Jinwei Miao, Department of Gynecologic Oncology, Beijing Obstetrics and Gynecology Hospital, Capital Medical University, 17 Qi He Lou Street, Beijing 100006, P.R. China E-mail: miaojinweigyn@163.com

Dr Jian Liu, Medical Research Center, Beijing Chao-Yang Hospital, Capital Medical University, 8 Gong Ti Nan Lu, Beijing 100020, P.R. China

E-mail: liujian2004811@126.com

Abbreviations: PUMA, p53 upregulated modulator of apoptosis; Ad-p53, adenovirus expressing p53; TAX, Taxol; DMSO, dimethyl sulfoxide; OD, optical density; RI, resistance index; FACS, fluorescence-activated cell sorting

Key words: ovarian cancer, Taxol resistance, p53 upregulated modulator of apoptosis, adenovirus expressing p53, gene therapy bution was identified. PUMA, a potent pro-apoptotic protein, was markedly suppressed in the SKOV3/TAX cells. Ad-p53 infection stimulated the upregulation of PUMA and re-sensitized the resistant ovarian cancer cells to TAX by an apoptotic mechanism. Therefore, Ad-p53 infection is an effective gene therapy method to re-sensitize the resistant ovarian cancer cells to TAX by restoring the expression of PUMA.

\section{Introduction}

Ovarian cancer is the most life-threatening gynecological malignancy, with a 5-year survival rate of $<30 \%$ (1-3). Since ovarian cancer is asymptomatic until the late stages, $\sim 70 \%$ patients are diagnosed with advanced stage (stage III/IV) $(4,5)$. Cytoreductive surgery, followed by intense chemotherapy with platinum and Taxol (TAX) is the current standard treatment for ovarian cancer (6).

TAX is a microtubule poison, it stabilizes microtubules and, as a result, causes defects in mitotic spindle assembly, chromosome segregation and cell division (7). The development of acquired drug resistance is the primary cause of chemotherapy failure in the treatment of ovarian cancer. The majority of patients with ovarian cancer show an excellent initial response to TAX therapy, however, this is followed by the eventual evolution of fatal drug resistance following multiple chemotherapeutic cycles.

Despite being investigated for decades, the molecular mechanism underlying acquired resistance to TAX in ovarian cancer cells remains to be elucidated, although one possible explanation is the dysregulation of apoptosis (8). p53 upregulated modulator of apoptosis (PUMA) is an important regulator of apoptosis and is involved in drug resistance. It is a BH3-only member belonging to the B-cell lymphoma 2 (Bcl-2) family and is important in mediating p53-dependent and p53-independent apoptosis by binding to anti-apoptotic Bcl-2 family members to induce mitochondrial dysfunction and the activation of caspase (9). PUMA is an important sensitizer of several types of cancer to chemotherapeutic agents. Introduction of PUMA significantly sensitizes the lung cancer cells to TAX, cisplatin, 5-fluorouracil and etoposide (10), while deletion of PUMA in human colorectal cancer cells results in resistance to apoptosis induced by adriamycin (11).

p53 is the most well-known transcriptional activator of PUMA, which binds directly to p53-responsive elements in the 
PUMA promoter (12). In the p53 apoptotic pathway, PUMA is the direct downstream effector of p53, with PUMA accounting for almost all the proapoptotic activity of p53 (9). In addition, targeted deletion of PUMA impairs the proapoptotic function of p53 (11). Delivery of p53 by Ad-p53 is a safe clinical method to activate the apoptosis of cancer cells and the injection of recombinant human Ad-p53 (Gendicine) has already been approved in the clinical treatment of several types of cancer in China $(13,14)$.

In the present study, to investigate the mechanism underlying the acquired resistance to TAX in ovarian cancer cells, a highly TAX-resistant ovarian cancer cell line SKOV3/TAX was induced and the differences in features compared with SKOV3 were examined.

\section{Materials and methods}

Establishment of the TAX-resistant ovarian cell line SKOV3/TAX. The human epithelial ovarian cancer cell line SKOV3 [American Type Culture Collection (ATCC), Manassas, VA, USA] was cultured in RPMI-1640 (Bioroc, Tianjin, China) supplemented with $10 \%$ fetal bovine serum (FBS) at $37^{\circ} \mathrm{C}$ in a $5 \% \mathrm{CO}_{2}$ standard incubator (Thermo Fisher Scientific, Inc., Waltham, MA, USA). The TAX-resistant ovarian cancer cell line SKOV3/TAX was established using a gradient concentration increment method. Each time TAX (Beijing SL Pharmaceutical Co., Ltd., Beijing,China) was added to the SKOV3 cells, the dead cells were discarded and the remaining viable cells were regarded as being drug-resistant. The remaining viable cells were then cultured in a higher concentration of TAX. With the gradual increase of TAX, SKOV3/TAX cells were able to survive in a final culture medium containing $0.1 \mu \mathrm{g} / \mathrm{ml} \mathrm{TAX}$. With stable resistance, the SKOV3/TAX cells were then cultured in a medium in the absence of any drug for 2-3 weeks prior to experiments.

MTT assay to evaluate the resistance index (RI) of SKOV3/TAX cells. SKOV3 and SKOV3/TAX cells $\left(8 \times 10^{3}\right)$ were seeded in a 96-well plate and incubated overnight. Various concentrations $(0.004,0.006,0.008,0.01,0.012$, $0.014,0.016,0.018$ and $0.02 \mu \mathrm{g} / \mathrm{ml} \mathrm{TAX}$ for SKOV3; 0.1 , $0.12,0.16,0.2,0.24,0.28,0.32,0.36$ and $0.4 \mu \mathrm{g} / \mathrm{ml} \mathrm{TAX}$ for SKOV3/TAX) of TAX were added to the RPMI-1640 with $10 \%$ FBS medium and the cells were incubated for $72 \mathrm{~h}$. The cells were incubated with MTT $(0.5 \mathrm{mg} / \mathrm{ml}$; Sigma-Aldrich, St. Louis, MO, USA) for $4 \mathrm{~h}$ at $37^{\circ} \mathrm{C}$, with $5 \% \mathrm{CO}_{2}$ atmosphere, and the formazan precipitate was then dissolved in $150 \mu \mathrm{l}$ dimethyl sulfoxide (DMSO; Sigma-Aldrich). The optical density (OD) values at $490 \mathrm{~nm}$ were measured using a microplate reader (Thermo Fisher Scientific, Inc.). The rates of inhibition were calculated according to the OD values. The $\mathrm{IC}_{50}$ was defined as the concentration of TAX required to reduce the optical density by $50 \%$ and RI was calculated to evaluate the drug resistance of SKOV3/TAX, which was defined as $\mathrm{IC}_{50} \mathrm{SKOV} 3 / \mathrm{TAX} / \mathrm{IC}_{50} \mathrm{SKOV} 3$.

Proliferation assay. Cells $\left(2 \times 10^{3}\right)$ were seeded into 96-well plates in triplicate and harvested on days 1,2,3, 4, 5 and 6. At the beginning of harvesting, MTT $(0.5 \mathrm{mg} / \mathrm{ml})$ was added to each well. Following incubation for $4 \mathrm{~h}$ at $37^{\circ} \mathrm{C}, 150 \mu \mathrm{l}$ DMSO was added to dissolve any formazan crystals that were present. The absorbance was measured at $490 \mathrm{~nm}$ using a microplate reader.

Flow cytometric analysis. Fluorescence-activated cell sorting (FACS) analysis was performed to assess the cell cycle distribution and identify the sub-G1 cells. Briefly, $72 \mathrm{~h}$ after treatment with or without Ad-p53 (50 MOI) followed by TAX incubation, the cells were collected (attached and floating) and fixed with $70 \%$ ethanol at $-20^{\circ} \mathrm{C}$ overnight. Following two washes with phosphate-buffered saline, the cells were treated with RNase A $(100 \mu \mathrm{g} / \mathrm{ml}$; Takara Biotechnology Co., Ltd., Dalian, China) at $37^{\circ} \mathrm{C}$ for $30 \mathrm{~min}$. Intracellular DNA was labeled with propidium iodide $(50 \mu \mathrm{g} / \mathrm{ml})$ and analyzed using a flow cytometer (BD FACSCanto II; BD Biosciences, Franklin Lakes, NJ, USA).

Cell adhesion assay. For the cell adhesion assay, $2 \times 10^{4}$ cells were plated in triplicate in 96-well plates coated with Matrigel (BD Biosciences) and incubated at $37^{\circ} \mathrm{C}$ for 10,20 , 30, 40, 50 and $60 \mathrm{~min}$. The floating cells were discarded and the adherent cells were quantified using MTT analysis.

Colony formation assay. SKOV3 and SKOV3/TAX cells were seeded at a density of 500 cells/well in a 6-well plate. Cells were allowed to grow for 14 days prior to staining with Crystal Violet (Sigma-Aldrich). All experiments were repeated at least three times and similar results were obtained in each trial.

Western blot analysis. Whole-cell protein extracted from diversely-treated cells were prepared and quantified using a BCA Protein assay kit (Sigma-Aldrich). Protein ( $80 \mu \mathrm{g} / \mathrm{lane})$ was denatured, resolved on $10 \%$ SDS-PAGE gels and semi-dry transferred at $12 \mathrm{~V}$ for $3 \mathrm{~h}$ onto nitrocellulose membranes (Bio-Rad, Hercules, CA, USA). The membranes were probed with primary antibody against PUMA (Sigma-Aldrich), p53 (Santa Cruz Biotechnology, Inc., Santa Cruz, CA, USA), poly ADP-ribose polymerase (PARP; Santa Cruz Biotechnology, Inc.) or $\beta$-actin (Sigma-Aldrich) overnight at $4^{\circ} \mathrm{C}$, followed by incubation with secondary goat anti-rabbit or anti-mouse polyclonal IgG (Santa Cruz Biotechnology, Inc.) conjugated by horseradish peroxidase and detected using an ECL kit (Applygen Technologies Inc., Peking, China).

Recombinant adenovirus expressing p53 (Ad-p53). The recombinant adenoviruses Ad-p53 were constructed using the Ad-Easy system (Agilent Technologies, Inc. Santa Clara, CA, USA) with green fluorescent protein (GFP). High-titer viruses were produced in HEK293 cells (ATCC) and purified using $\mathrm{CsCl}_{2}$ gradient ultracentrifugation. Adenoviruses expressing GFP (Ad-GFP) were used as control adenoviruses in the present study.

Ad-p53 infection and drug resistance reversal analysis. To analyze the drug resistance reversal effect by Ad-p53, the cells were initially infected with Ad-p53 at a multiplicity of infection of 50 for $24 \mathrm{~h}$. Following this, a medium containing $10 \%$ FBS and TAX were added to the wells. The cells and 
TAX were co-cultured for another $48 \mathrm{~h}$ prior to further analysis, including FACS analysis of the sub-G1 proportion, western blot analysis to detect PUMA and cleaved-PARP and MTT analysis to measure cell survival.

Statistical analysis. Statistical analysis was performed using SPSS 10.0 software (SPSS, Inc., Chicago, IL, USA). The values in the figures are presented as the mean \pm standard deviation. $\mathrm{P}$ values were calculated by Student's t-test or two-way analysis of variance. $\mathrm{P}<0.05$ was considered to indicate a statistically significant difference.

Ethical Approval. The present study was approved by the Ethics Committee of Beijing Chao-Yang Hospital (Capital Medical University, Beijing, China).

\section{Results}

SKOV3/TAX cells are highly resistant to TAX. The TAX-resistant ovarian cancer cell line SKOV3/TAX was induced using the gradient concentration increment method and its capacity to resist TAX was measured using an MTT assay (data not shown). The result demonstrated that the induced SKOV3/TAX cell line was highly resistant to TAX, with the RI reaching 88.5. Successful construction of SKOV3/TAX provided a good cell model to characterize the TAX-resistant ovarian cancer cells and to examine the mechanism underlying TAX resistance.

SKOV3/TAX cells proliferate slower than SKOV3 cells. The present study compared the proliferation rate between SKOV3/TAX and SKOV3 cells using MTT analysis. The curve of cell proliferation revealed that the growth of the SKOV3/TAX cells was significantly slower than that of the SKOV3 cells (Fig. 1).

SKOV3/TAX possesses a higher colony-forming capacity. Although SKOV3/TAX proliferates at a slower rate than SKOV3, SKOV3/TAX possesses a higher colony-forming capacity. As shown in Fig. 2, when 500 cells were seeded, SKOV3/TAX formed $340 \pm 26$ colonies, while SKOV3 formed only $275 \pm 8$ and the colony-forming efficiency was $68 \pm 7.6$ and $55 \pm 2.8 \%$, respectively $(\mathrm{P}<0.05)$.

Adhesion ability is enhanced in SKOV3/TAX cells. Adhesion ability was also compared using MTT analysis. As shown in Fig. 3, at 10, 20 and 30 min after seeding, the adhesion proportion of SKOV3/TAX was markedly higher than the SKOV3 cells (all $\mathrm{P}<0.05$ ), indicating that the SKOV3/TAX cells exhibited a higher adhesion ability.

SKOV3/TAX shares a similar cell cycle distribution to SKOV3 without TAX treatment. The cell cycle distribution pattern was then compared between SKOV3/TAX and SKOV3 cells without TAX treatment. The result demonstrated a similar distribution pattern between the two (Fig. 4A and B), indicating that the cell cycle was not affected in SKOV3/TAX cells.

SKOV3/TAX cells are highly resistant to the inhibition of mitosis caused by TAX. TAX is a mitotic inhibitor used in

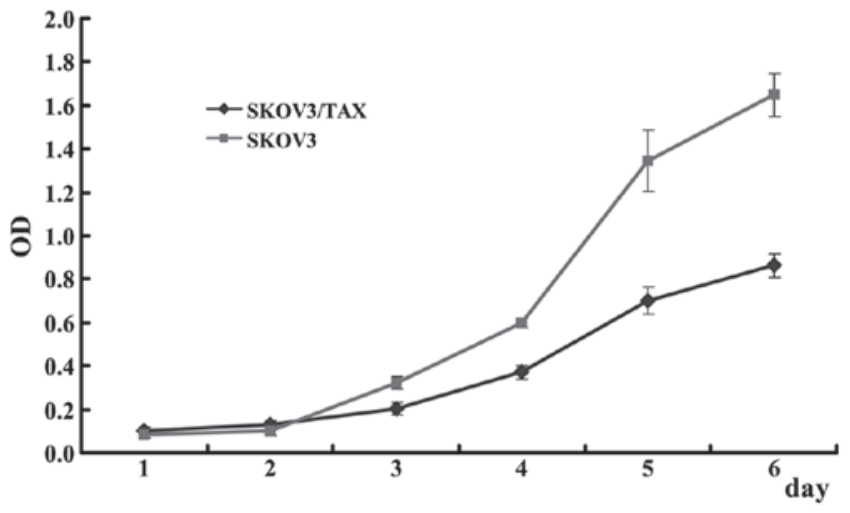

Figure 1. Proliferation of SKOV3/TAX cells was significantly slower than that of SKOV3 cells revealed by the cell growth curve. OD, optical density; TAX, Taxol.
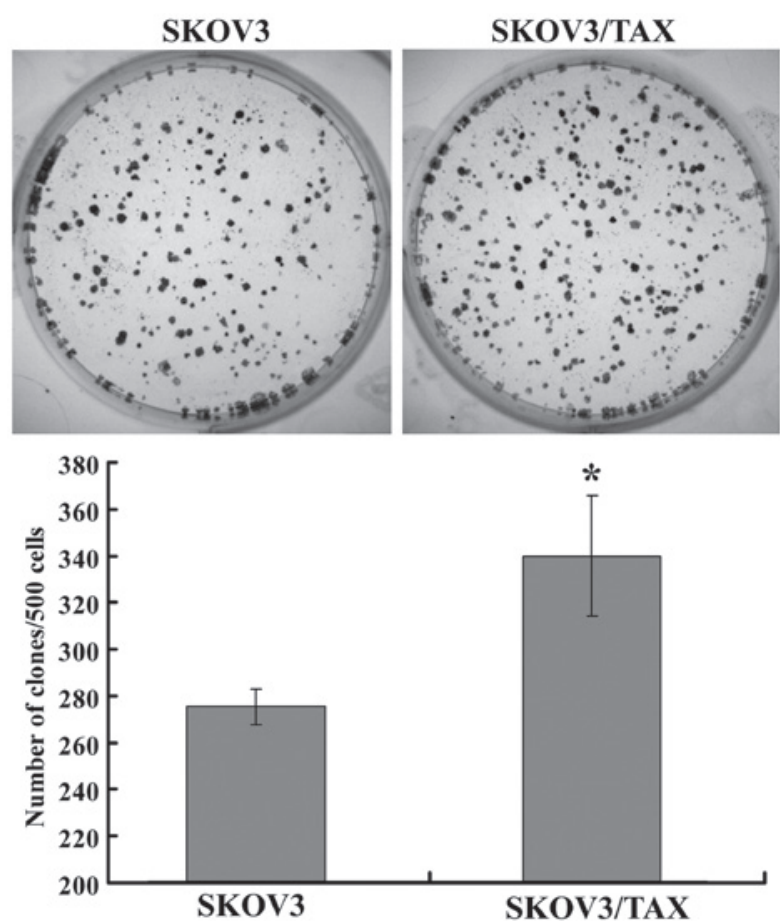

Figure 2. SKOV3/TAX forms more colonies than SKOV3. SKOV3/TAX possessed a higher colony-forming capacity than SKOV3. ${ }^{~} \mathrm{P}<0.05$ when the SKOV3/TAX group is compared with the SKOV3 group. TAX, Taxol.

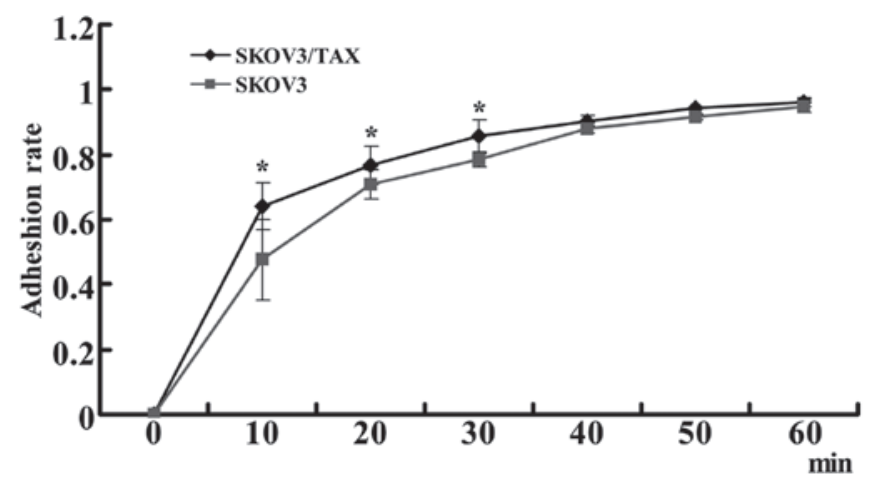

Figure 3. Cell adhesion ability of SKOV3/TAX and SKOV3 cells. The adhesion rate of SKOV3/TAX was significantly higher than SKOV3 10, 20 and 30 min after being seeded (" $\mathrm{P}<0.05$ when the SKOV3/TAX group is compared with the SKOV3 group). TAX, Taxol. 
A

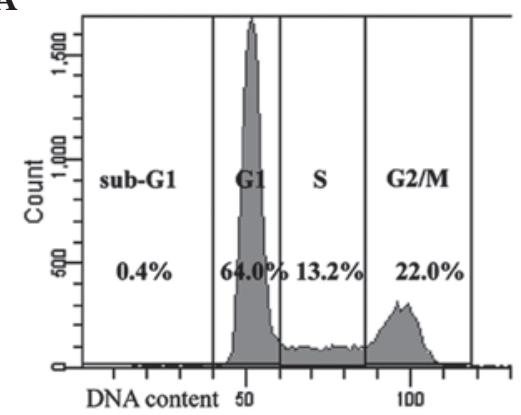

B

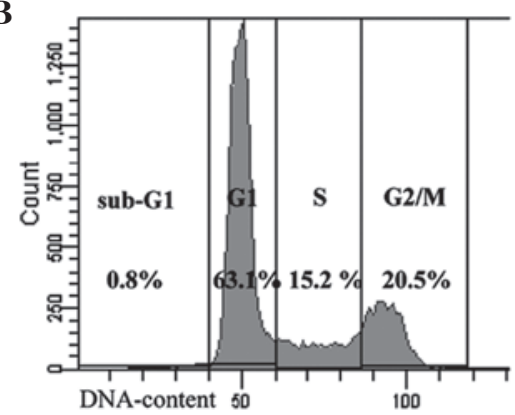

C
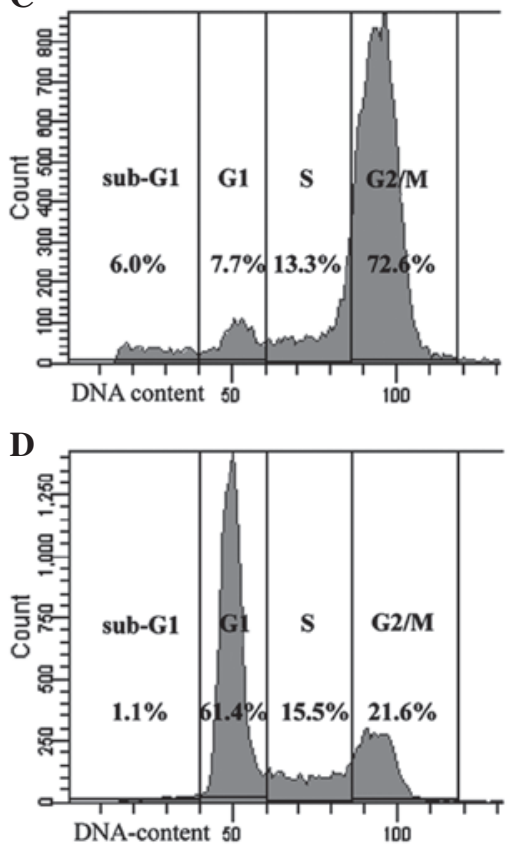

Figure 4. Cell cycle distribution pattern of SKOV3 and SKOV3/TAX cells with or without TAX treatment. Without TAX treatment, (A) SKOV3 and (B) SKOV3/TAX shared a similar pattern of cell cycle distribution. Following treatment with TAX (0.1 $\mu \mathrm{g} / \mathrm{ml})$, (C) SKOV3 was clearly inhibited at mitosis, while (D) SKOV3/TAX was not. TAX, Taxol.

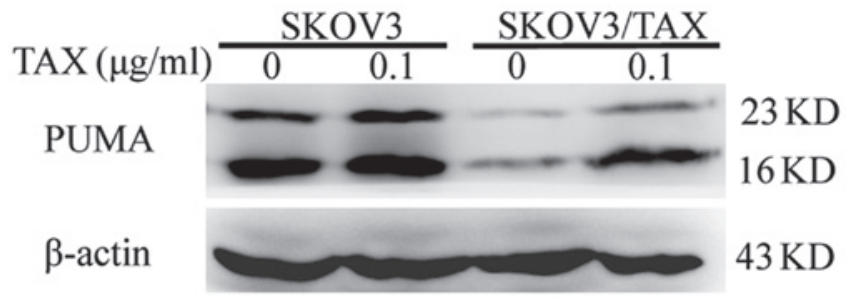

Figure 5. PUMA is downregulated in SKOV3/TAX cells. The expression of PUMA in SKOV3/TAX and SKOV3 cells with and without TAX treatment was detected by western blot analysis. TAX, Taxol; PUMA, p53 upregulated modulator of apoptosis.

cancer chemotherapy, including ovarian, breast and non-small cell lung cancer. It can arrest cells in mitosis through microtubule stabilization and induce apoptosis $(15,16)$. When treated with $0.1 \mu \mathrm{g} / \mathrm{ml}$ TAX for $24 \mathrm{~h}, \mathrm{SKOV} 3$ was markedly inhibited at the $\mathrm{G} 2 / \mathrm{M}$ phase (between 22.0 and $72.6 \%$ ) with a clear increase in late-apoptotic cells (between 0.4 and 6.0\%; Fig. 4A and C). However, no significant inhibition of mitosis (between 20.5 and $21.6 \%$ ) or late-apoptotic cells (between 0.8 and $1.1 \%$ ) was identified in the TAX-treated SKOV3/TAX cells (Fig. 4B and D).

PUMA is downregulated in SKOV3/TAX cells. To examine the mechanism underlying the TAX-resistance of ovarian cancer, the expression of PUMA was detected using western blot analysis. PUMA was markedly downregulated in the SKOV3/TAX cells compared with the control SKOV3 in the presence or absence of TAX treatment (Fig. 5). PUMA is a potent pro-apoptotic gene that contributes to the drug resistance of multiple types of cancer. The inhibition of PUMA disrupts the p53-dependent and p53-independent apoptotic pathway.
Ad-p53 infection stimulates suppressed PUMA in SKOV3/TAX cells and restores the apoptotic response of SKOV3/TAX cells to TAX. PUMA is the direct transcriptional downstream gene of p53. In the present study, to restore the expression of PUMA in SKOV3/TAX cells, p53 was upregulated by infection with Ad-p53. The results confirmed that Ad-p53 infection stimulated the expression of PUMA, in the presence or absence of TAX treatment. Ad-p53 was used in the present study due to two main advantages, its powerful ability to activate the transcription of PUMA and, importantly, its confirmed safety for use in the clinic.

The present study then examined whether the recovery of PUMA by Ad-p53 was able to restore the apoptotic response of SKOV3/TAX cells to TAX. As shown in Fig. 6A, sub-G1 measurement by FACS analysis revealed that treatment of the SKOV3/TAX cells with either Ad-p53 or TAX alone led to only a small proportion of late apoptotic cells (1.3 and 7.2\%, respectively), while combinational treatment with Ad-p53 and TAX resulted in a significantly higher proportion of late apoptotic cells $(23.6 \%)$, which was $\sim 3$ fold that of the total effect of treatment with Ad-p53 or TAX alone. The same result was also achieved by measurement of cleaved-PARP, which is a specific molecular marker of apoptotic cells. As shown in Fig. 6B and C, the quantity of cleaved-PARP produced by the combination of Ad-p53 and TAX was significantly higher than that of Ad-p53 or TAX alone (also 3-fold). Collectively, these results clearly indicated that Ad-p53 infection restored the apoptotic response of SKOV3/TAX cells to TAX.

Ad-p53 reverses the resistance of SKOV3/TAX cells to TAX. The inhibition of apoptosis is a major approach used by drug-resistant cancer cells to survive following drug treatment. Following the observation that infection with Ad-p53 
A
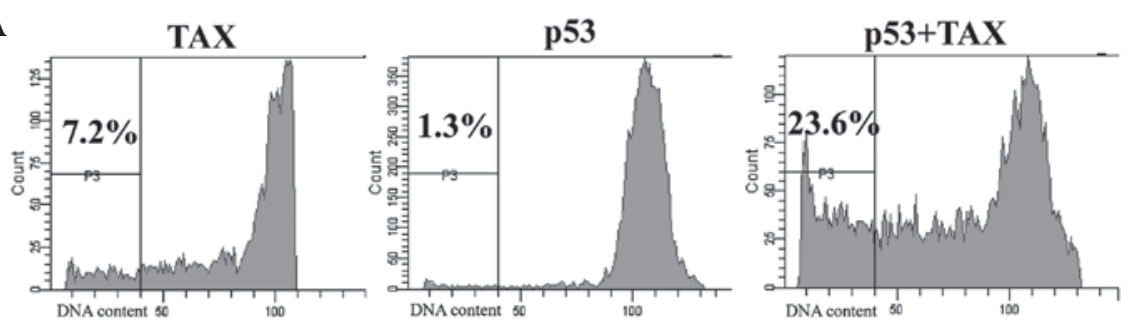

B

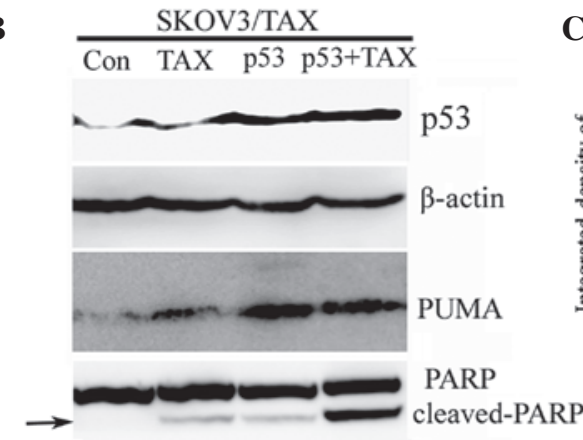

C

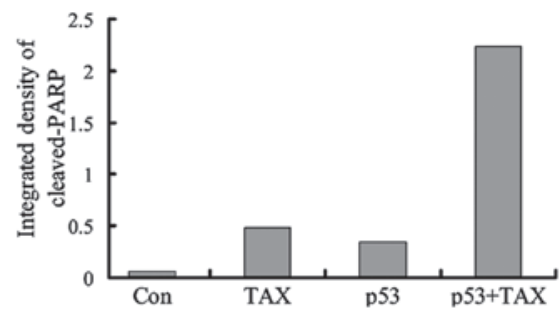

Figure 6. Ad-p53 restores the apoptotic response of SKOV3/TAX to TAX. TAX $(0.4 \mu \mathrm{g} / \mathrm{ml})$ and adenoviruses with an MOI of 50 were used in this experiment. For the control group, the SKOV3/TAX cells were grown in medium for $72 \mathrm{~h}$ without treatment. For the TAX group, the SKOV3/TAX cells were initially infected with a $50 \mathrm{MOI}$ control adenovirus (Ad-GFP) for $24 \mathrm{~h}$ and then incubated with $0.4 \mu \mathrm{g} / \mathrm{ml}$ TAX for another $48 \mathrm{~h}$. For the p53 group, the SKOV3/TAX cells were first infected with 50 MOI Ad-p53 for $24 \mathrm{~h}$ and then added with the same volume of medium without TAX. For the p53 + TAX group, the SKOV3/TAX cells were initially infected with 50 MOI Ad-p53 for $24 \mathrm{~h}$ and then incubated with $0.4 \mu \mathrm{g} / \mathrm{ml}$ TAX for another $48 \mathrm{~h}$. Each group of cells were collected at $72 \mathrm{~h}$ and then analyzed by (A) FACS and (B) western blot analysis. (C) The integrated density of cleaved-PARP was measured by Image J software. Con, control; Ad-p53, adenovirus expressing p53; Ad-GFP, adenovirus expressing green fluorescent protein; TAX, Taxol; PARP, poly ADP-ribose polymerase; FACS, fluorescence-activated cell sorting; MOI, multiplicity of infection.

A

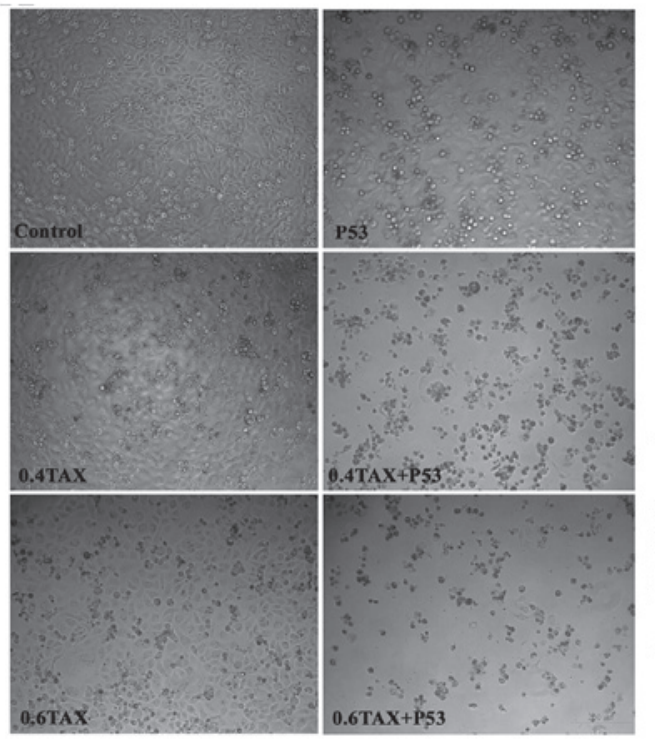

B

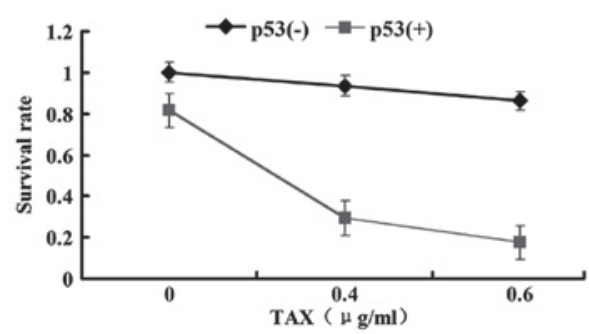

C

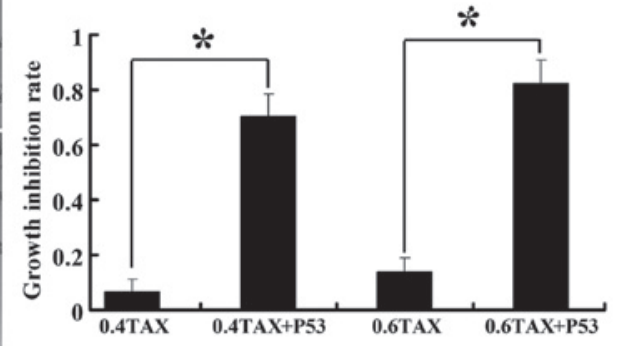

Figure 7. Ad-p53 re-sensitizes SKOV3/TAX cells to TAX. (A) Visualization of the effect of Ad-p53 on re-sensitizing SKOV3/TAX cells to TAX (0.4 and $0.6 \mu \mathrm{g} / \mathrm{ml}$ ) under a microscope. (B) Survival rate analysis indicated that infection with Ad-p53 markedly re-sensitizes SKOV3/TAX cells to TAX. (C) On treatment with $0.4 \mu \mathrm{g} / \mathrm{ml}$ TAX, Ad-p53 enhanced the sensitivity of SKOV3/TAX to TAX by 10 -fold, and on treatment with $0.6 \mu \mathrm{g} / \mathrm{ml}$ TAX, Ad-p53 enhanced the sensitivity by 6 -fold. " $\mathrm{P}<0.05$ when the SKOV3/TAX group is compared with the SKOV3 group. Ad-p53, adenovirus expressing p53; TAX, Taxol.

upregulated the expression of the suppressed PUMA in SKOV3/TAX cells and restored the apoptotic response to TAX, the present study aimed to determine whether Ad-p53 can reverse the resistance of SKOV3/TAX cells to TAX.

The effect of Ad-p53 on re-sensitizing the resistant cells to TAX was measured using MTT analysis and visualized by microscopy (magnification, x10; BX51, Olympus, Tokyo, Japan). The pro-apoptotic and growth inhibition effects of Ad-p53 were first revealed by direct visualization under a microscope ( Fig. 7A). Furthermore, as shown in Fig. 7B, the survival curve reveals that following treatment with TAX, infection with Ad-p53 caused a significant reduction in the 
survival of the resistant cells. The sensitivity of SKOV3/TAX to TAX was significantly improved by 10 -fold and 6 -fold following treatment with 0.4 and $0.6 \mu \mathrm{g} / \mathrm{ml} \mathrm{TAX}$, respectively (Fig. 7C).

\section{Discussion}

Ovarian cancer is the main cause of mortality in gynecological malignancy. Surgery and chemotherapy have been used to treat human ovarian cancer, however, the long-term survival rate remains unsatisfactory due to eventual tumor recurrence and the development of chemotherapy resistance. TAX, which acts by inducing cell cycle arrest and inhibiting cell proliferation (17), is one of the most widely used chemotherapeutic agents in the treatment of ovarian cancer. However, its therapeutic value in ovarian cancer is also largely restricted by TAX-resistance acquired during treatment, the underlying mechanism of which remains to be elucidated.

In vitro study is an important approach used in the investigation of drug resistance. In the present study, the gradient increase of TAX concentration in parental SKOV3 cells induced a highly TAX-resistant cell line, SKOV3/TAX. The resistance of this cell line was stable for long-term examination.

Several properties of the ovarian cancer cells altered during the acquirement of TAX resistance. Compared with the control cells, the TAX-resistant ovarian cancer cells were characterized by a marked increase in resistance to TAX-induced mitotic arrest and apoptosis, a significantly slower proliferation rate, increased colony-forming efficiency and an increased adhesion ability. However, no significant difference was observed in cell cycle distribution. These unique features, including a lower proliferation rate, may be useful in differentiating TAX-resistant ovarian cancer from TAX-sensitive ovarian cancer in order to target them for specialized therapy.

It has been revealed that deregulated apoptosis contributes to the development of acquired resistance to chemotherapeutic agents (18). Apoptosis, also termed programmed cell death, is a genetically encoded program that eliminates unwanted cells. Defects in one or more components of the apoptotic pathway may result in resistance to drug-induced apoptosis (19). In the present study, detection revealed that the proapoptotic protein PUMA was suppressed in the induced TAX-resistant ovarian cancer cells. PUMA is an important apoptotic sensitizer of various types of cancer to chemotherapeutic drugs $(11,20)$.

Genetic restoration of the apoptotic pathway or introduction of proapoptotic molecules is an attractive approach to destroy drug-resistant cancer cells. In the p53 apoptotic pathway, PUMA is the direct downstream proapoptotic effector of p53. The present study demonstrated that delivery of p53 into SKOV3/TAX by Ad-p53 re-upregulated the expression of PUMA and restored the apoptotic response to TAX, which was indicated by sub-G1 analysis and cleaved-PARP detection. It was also confirmed that the sensitivity of SKOV3/TAX to $0.4 \mu \mathrm{g} / \mathrm{ml}$ TAX treatment was improved up to 10 -fold. Ad-p53 was used in the present study due to its high efficiency in activating the transcription of PUMA and, importantly due to its established approval for clinical use. The injection of recombinant human Ad-p53, with the brand name Gendicine, is the first gene therapy product to be approved for clinical use in humans in China $(13,14)$. This virus is designed to treat patients with types of tumor in which p53 genes are mutated.

Resistance to TAX remains a major obstacle in the successful treatment of ovarian cancer. The present study demonstrated that combination therapy of TAX with Ad-p53 is an effective approach to reverse TAX resistance in ovarian cancer in vitro. Considering the confirmed clinical safety of Ad-p53, this treatment method may also be valuable in the future clinical treatment of drug-resistant, metastatic or locally recurrent ovarian cancer.

\section{Acknowledgements}

This study was financially supported by grants from the Beijing Natural Science Foundation (no. 7102056).

\section{References}

1. Rankin EB, Fuh KC, Taylor TE, et al: AXL is an essential factor and therapeutic target for metastatic ovarian cancer. Cancer Res 70: 7570-7579, 2010.

2. Wang C,Zhou L, Li S, et al: C4orf7 contributes to ovarian cancer metastasis by promoting cancer cell migration and invasion. Oncol Rep 24: 933-939, 2010

3. Gubbels JA, Claussen N, Kapur AK, Connor JP and Patankar MS: The detection, treatment, and biology of epithelial ovarian cancer. J Ovarian Res 3: 8, 2010.

4. Tillmanns TD, Lowe MP, Walker MS, Stepanski EJ and Schwartzberg LS: Phase II clinical trial of bevacizumab with albumin-bound paclitaxel in patients with recurrent, platinum-resistant primary epithelial ovarian or primary peritoneal carcinoma. Gynecol Oncol 128: 221-228, 2013.

5. Jemal A, Siegel R, Ward E, et al: Cancer statistics, 2006. CA Cancer J Clin 56: 106-130, 2006.

6. Heintz AP, Odicino F, Maisonneuve P, et al: Carcinoma of the ovary. FIGO 26th annual report on the results of treatment in gynecological cancer. Int J Gynaecol Obstet 95: S161-S192, 2006.

7. Mollinedo F and Gajate C: Microtubules, microtubule-interfering agents and apoptosis. Apoptosis 8: 413-450, 2003.

8. Kigawa J: New strategy for overcoming resistance to chemotherapy of ovarian cancer. Yonago Acta Med 56: 43-50, 2013.

9. Yu J and Zhang L: PUMA, a potent killer with or without p53. Oncogene 27: S71-S83, 2008.

10. Yu J, Yue W, Wu B and Zhang L: PUMA sensitizes lung cancer cells to chemotherapeutic agents and irradiation. Clin Cancer Res 12: 2928-2936, 2006.

11. Yu J, Wang Z, Kinzler KW, Vogelstein B and Zhang L: PUMA mediates the apoptotic response to $\mathrm{p} 53$ in colorectal cancer cells. Proc Natl Acad Sci USA 100: 1931-1936, 2003.

12. Chipuk JE, Bouchier-Hayes L, Kuwana T, Newmeyer DD and Green DR: PUMA couples the nuclear and cytoplasmic proapoptotic function of p53. Science 309: 1732-1735, 2005.

13. Peng Z: Current status of gendicine in China: recombinant human Ad-p53 agent for treatment of cancers. Hum Gene Ther 16: 1016-1027, 2005.

14. Pearson S, Jia $\mathrm{H}$ and Kandachi K: China approves first gene therapy. Nat Biotechnol 22: 3-4, 2004.

15. Long BH and Fairchild CR: Paclitaxel inhibits progression of mitotic cells to G1 phase by interference with spindle formation without affecting other microtubule functions during anaphase and telephase. Cancer Res 54: 4355-4361, 1994.

16. Schiff PB, Fant J and Horwitz SB: Promotion of microtubule assembly in vitro by taxol. Nature 277: 665-667, 1979.

17. Horwitz SB: Taxol (paclitaxel): mechanisms of action. Ann Oncol 5: S3-S6, 1994.

18. Johnstone RW, Ruefli AA and Lowe SW: Apoptosis: a link between cancer genetics and chemotherapy. Cell 108: 153-164, 2002.

19. Fisher DE: Apoptosis in cancer therapy: crossing the threshold. Cell 78: 539-542, 1994.

20. Wang H, Qian H, Yu J, et al: Administration of PUMA adenovirus increases the sensitivity of esophageal cancer cells to anticancer drugs. Cancer Biol Ther 5: 380-385, 2006. 